\title{
Estimation of internal pressure of liquids and liquid mixtures
}

\author{
N. Santhi ${ }^{1}$, P. Sabarathinam ${ }^{2}$, G. Alamelumangai ${ }^{1}$, \\ J. Madhumitha ${ }^{1}$, M. Emayavaramban ${ }^{1}$ \\ 1 Department of Chemistry, Government Arts College, C.Mutlur, Chidambaram-608102, India \\ ${ }^{2}$ Retd. Registrar \& Head of the Department of Technology, Annamalai University, \\ Annamalainagar-608002, India \\ E-mail address: nsaanthi@gmail.com
}

\begin{abstract}
By combining the van der Waals' equation of state and the Free Length Theory of Jacobson, a new theoretical model is developed for the prediction of internal pressure of pure liquids and liquid mixtures. It requires only the molar volume data in addition to the ratio of heat capacities and critical temperature. The proposed model is simple, reliably accurate and capable of predicting internal pressure of pure liquids with an average absolute deviation of $4.24 \%$ in the predicted internal pressure values compared to those given in literature. The average absolute deviation in the predicted internal pressure values through the proposed model for the five binary liquid mixtures tested varies from $0.29 \%$ to $1.9 \%$ when compared to those of literature values.
\end{abstract}

\section{Keywords:}

van der Waals; pressure of liquids; theory of Jacobson; capacity of heat liquids; equation of Srivastava and Berkowitz

\section{INTRODUTION}

The importance of internal pressure in understanding the properties of liquids and the full potential of internal pressure as a structural probe did become apparent with the pioneering work of Hildebrand ${ }^{1,2}$ and the first review of the subject by Richards ${ }^{3}$ appeared in 1925. In an excellent article Dack ${ }^{4}$ reviewed the importance of solvent internal pressure and cohesion to solution phenomena. A review on the relationship between the intermolecular forces and properties of solution was made by Kortum ${ }^{5}$.

Barton $^{6}$ discussed the relationship between internal pressure and molar volume in some depth. Pointing out that internal pressure is a uniquely important parameter in sonochemistry, Srivastava and Berkowitz ${ }^{7}$ developed an expression, which permits the evaluation of internal pressure from readily available and easily measurable quantities. In this work a simple equation is proposed by incorporating the Free Length Theory of Jacobson ${ }^{8,9}$ and van der Waals' equation of state; the proposed model is a purely predictive tool for predicting internal pressure of liquids and liquid mixtures. Computed internal pressure values for liquids and liquid mixtures through the proposed equation are compared with those of literature. 


\section{PREVIOUS WORK}

Starting from the van der Waals equation of state

$$
\mathrm{P}+\left(\mathrm{a} / \mathrm{V}^{2}\right)=\mathrm{RT} /(\mathrm{V}-\mathrm{b})
$$

With $\mathrm{P}=$ pressure

$\mathrm{V}=$ molar volume

$\mathrm{R}=$ universal gas constant

$\mathrm{T}=$ temperature

$\mathrm{a}$ and $\mathrm{b}=$ van der Waals' constants,

Srivastava and Berkowitz ${ }^{7}$ arrived at

$$
u^{2} \rho=\left({ }_{L}^{\gamma} b / R T\right) \pi_{i}^{2}
$$

where $\mathrm{u}=$ ultrasonic velocity

$\rho=$ density

$\gamma_{L}=$ ratio of heat capacities of liquid

And $\pi_{\mathrm{i}}=$ internal pressure

Replacing $b$, in terms of $M$ and $\rho$, in eqn. [1] they obtained an expression for internal pressure of a liquid:

$$
\pi_{\mathrm{i}}=\mathrm{u \rho} / \mathrm{M}^{1 / 2} \mathrm{k}
$$

Where $\mathrm{M}=$ molar mass of liquid

Present Work

1. Estimation of Internal Pressure using the Srivastava - Berkowitz equation

Having fitted the experimental $\pi_{\mathrm{i}}$, $\mathrm{u}$ and $\rho$ values in Eqn. (2), Srivastava and Berkowitz obtained the constant $(\mathrm{k})$ values for a few families of organic compounds. The reported $\mathrm{k}_{\mathrm{SB}}$ (known as Srivastava and Berkowitz constant) values directly yield $\pi_{\mathrm{i}}$ values in atmospheres, provided $u$ and $\rho$ are in c.g.s. system of units. When one is to use SI system of units.

or

$$
\begin{array}{ll}
\pi_{\mathrm{i}}=\mathrm{u} \rho / 10 \mathrm{k}_{\mathrm{SB}} \mathrm{M}^{1 / 2} & \text { (in atm) } \\
\pi_{\mathrm{i}}=10^{3} \times 101.325\left[\mathrm{u} \rho /\left(10 \mathrm{k}_{\mathrm{SB}} \mathrm{M}^{1 / 2}\right)\right] & \text { (in Pa) }
\end{array}
$$

2. Internal Pressure from $u, \rho$ and ${ }^{\gamma} \underline{L}$

Consider Eqn. (1)

$$
\mathrm{u}^{2} \rho=\left({ }_{\mathrm{L}} \mathrm{b} / \mathrm{RT}\right) \pi_{\mathrm{i}}^{2}
$$

Approximating that $\mathrm{b} \approx \mathrm{M} / 4 \rho$ and simplifying, Eqn. (1) becomes

$$
\pi_{\mathrm{i}}=2 \mathrm{u \rho}(\mathrm{RT})^{1 / 2} /\left({ }_{\mathrm{L}} \mathrm{M}\right)^{1 / 2}
$$


This can be shown to be equal to

$$
\begin{aligned}
\pi_{\mathrm{i}} & =(1 / 555.613)\left(\mathrm{u} \rho / \mathrm{M}^{1 / 2}\right)\left(\mathrm{T} / \gamma_{\mathrm{L}}\right)^{1 / 2} \\
\text { i.e., } \quad \pi_{\mathrm{i}} & =\left(1.7998 \times 10^{-3}\right)\left(\mathrm{u} \rho / \mathrm{M}^{1 / 2}\right)\left(\mathrm{T} / \gamma_{\mathrm{L}}\right)^{1 / 2} \quad \text { (in atm) } \\
\text { or } \quad \pi_{\mathrm{i}} & =182.3656\left(\mathrm{u} \rho / \mathrm{M}^{1 / 2}\right)\left(\mathrm{T} / \gamma_{\mathrm{L}}\right)^{1 / 2} \quad \text { (in Pa) }
\end{aligned}
$$

(Note $u$ and $\rho$ are in SI units)

Eqns. (5) and (6) are useful in predicting internal pressures when $\mathrm{k}_{\mathrm{SB}}$ values are not available and when $u, \rho$ and ${ }_{L}^{\gamma}$ are known, Eqn. (5) and (6) describe $\mathrm{k}_{\mathrm{SB}}$ in terms ${ }_{\mathrm{L}}$ and T. For example from Eqn. (5) one can show that

$$
\mathrm{k}_{\mathrm{SB}} \text { in SI units }=555.613\left({ }_{\mathrm{L}}^{\gamma} / \mathrm{T}\right)^{1 / 2}
$$

3. New Model Development

$$
\mathrm{u}^{2} \rho=\left({ }_{\mathrm{L}}^{\gamma} \mathrm{b} / \mathrm{RT}\right) \pi_{\mathrm{i}}^{2}
$$

This is the starting point for developing a new expression here. It can be written as

$$
\pi_{\mathrm{i}}=\mathrm{u} \rho^{1 / 2} /\left({ }_{\mathrm{L}}^{\gamma} \mathrm{b} / \mathrm{RT}\right)^{1 / 2}
$$

Jacobson's ${ }^{8}$ definition for free length is

$$
\mathrm{L}_{f}=2 \mathrm{~V}_{\mathrm{a}} / \mathrm{Y}
$$

where $\mathrm{V}_{\mathrm{a}}=$ available molar volume $=\mathrm{V}-\mathrm{V}_{0}$

$\mathrm{V}=$ molar volume at a given temperature

$\mathrm{V}_{0}=$ molar volume at absolute zero temperature $(0 \mathrm{~K})$

and $\quad \mathrm{Y}=$ molar surface area of the molecules $=\left(36 \pi \mathrm{NV}_{0}^{2}\right)^{1 / 3}$

According to Jacobson's free length theory, free length, $\mathrm{L}_{f}$ and adiabatic compressibility, $\beta\left(=1 / \mathrm{u}^{2} \rho\right)$ are related by

$$
\mathrm{L}_{f} / \beta^{1 / 2}=\left(\mathrm{u}^{2} \rho\right)^{1 / 2} \mathrm{~L}_{f}=\mathrm{u} \rho^{1 / 2} \mathrm{~L}_{f}=\mathrm{K}
$$

$\mathrm{K}$, in Eqn. (10) is the Jacobson constant and it is temperature dependent. Replacing $\mathrm{L}_{f}$ in Eqn.(10) by molar volumes and molar surface area of the molecules as per Eqn. (9), Eqn. (10) can be written as

$$
\mathrm{u} \rho^{1 / 2}=\mathrm{K} Y / 2 \mathrm{~V}_{\mathrm{a}}
$$

In the light of Eqn. (11), Eqn. (8) turns out to

$$
\pi_{\mathrm{i}}=\mathrm{K} \mathrm{Y} / 2 \mathrm{~V}_{\mathrm{a}}\left({ }_{\mathrm{L}}^{\gamma} \mathrm{b} / \mathrm{RT}\right)^{1 / 2}
$$

Approximating that

$$
\mathrm{b} \approx \mathrm{V} / 4
$$


Eqn. (12) reduces to

$$
\pi_{\mathrm{i}}=\mathrm{K} \mathrm{Y} / 2 \mathrm{~V}_{\mathrm{a}} \mathrm{V}^{1 / 2}\left({ }_{\mathrm{L}}^{\gamma} / 4 \mathrm{RT}\right)^{1 / 2}=\mathrm{K} \mathrm{Y} / 2 \mathrm{~V}_{\mathrm{a}} \mathrm{V}^{1 / 2} \mathrm{k}_{\mathrm{SBSI}}, \quad\left(\text { in } \mathrm{kg} / \mathrm{ms}^{2}\right)
$$

With $\mathrm{k}_{\mathrm{SBSI}}=\left({ }_{\mathrm{L}}^{\gamma} / 4 \mathrm{RT}\right)^{1 / 2},\left(\right.$ in $\left.(\mathrm{kgmol} / \mathrm{kg})^{1 / 2} \mathrm{~s} / \mathrm{m}\right)$

It can be shown that

$\left.\mathrm{k}_{\mathrm{SBSI}}=555.613\left({ }_{\mathrm{L}}^{\gamma} \mathrm{T}\right)^{1 / 2},(\text { in }(\mathrm{kg} \mathrm{kgmol}))^{1 / 2} / \mathrm{m}^{2} \mathrm{~s} \mathrm{~atm}\right)$

$$
=10 \mathrm{k}_{\mathrm{SB}}
$$

Eqn. (14) is the new equation proposed in this work for the estimation of internal pressure from pure component properties, particularly, from molar volumes. As derived, the new equation is the result of a combination of the van der Waals' equation of state and the Free Length Theory of Jacobson ${ }^{8,9}$.

The various quantities required to use Eqn. (14) are described below

$$
\begin{aligned}
& \mathrm{K}=\mathrm{KJ} \times 10^{-10},(\mathrm{~m} \mathrm{~kg})^{1 / 2} / \mathrm{s} \\
& \mathrm{K}_{\mathrm{J}}=\text { Jacobson's constant in } \mathrm{kg}^{1 / 2}\left(\mathrm{~A}^{0}\right) / \mathrm{m}^{1 / 2} \mathrm{~s}=18687+40.391 \mathrm{t}\left({ }^{\circ} \mathrm{C}\right) \text { (Reference 10) } \\
& \mathrm{Y}=\left(36 \pi \mathrm{NV}_{0}^{2}\right)^{1 / 3}=\left[36 \pi\left(6.023 \times 10^{26}\right)\right]^{1 / 3} \mathrm{~V}_{0}{ }^{2 / 3} \\
& \left.=4.084 \times 10^{9} \mathrm{~V}_{0}^{2 / 3} \text {, (in } \mathrm{m}^{2} / \mathrm{kg} \mathrm{mol}\right), \mathrm{V}_{0} \text { in } \mathrm{m}^{3} / \mathrm{kg} \mathrm{mol} \\
& \mathrm{V}_{0}=\mathrm{V}\left[1-\left(\mathrm{T} / \mathrm{T}_{\mathrm{c}}\right)\right]^{0.3}=\mathrm{V}\left(1-\mathrm{T}_{\mathrm{r}}\right)^{0.3}, \quad \mathrm{~V} \text { in } \mathrm{m}^{3} / \mathrm{kg} \mathrm{mol} \\
& \mathrm{V}_{\mathrm{a}}=\mathrm{V}-\mathrm{V}_{0}, \quad \mathrm{~V}_{\mathrm{a}} \text { in } \mathrm{m}^{3} / \mathrm{kg} \mathrm{mol}
\end{aligned}
$$

And $\mathrm{R}=8314.3$, (in $\mathrm{kg} \mathrm{m}^{2} / \mathrm{kg} \mathrm{mol} \mathrm{s}^{2} \mathrm{~K}$ )

With the above Eqn. (14) is

$$
\pi_{\mathrm{i}}=\left(\mathrm{K}_{\mathrm{J}} \times 10^{-10}\right)\left(4.084 \times 10^{9}\right) \mathrm{V}_{0}^{2 / 3}(8314.3)^{1 / 2} / \mathrm{V}_{\mathrm{a}} \mathrm{V}^{1 / 2}\left({ }_{\mathrm{L}}^{\gamma} / \mathrm{T}\right)^{1 / 2}\left(\text { in } \mathrm{kg} / \mathrm{ms}^{2}\right)
$$

Eqn. (15) in terms of $\mathrm{K}, \mathrm{k}, \mathrm{Tc}$ and $\mathrm{V}$ turns to

$$
\pi_{\mathrm{i}}=37.239 \mathrm{~K}_{\mathrm{J}}\left(1-\mathrm{T}_{\mathrm{r}}\right)^{0.2} \mathrm{~T}^{1 / 2} / \mathrm{V}^{5 / 6}\left[1-(1-\mathrm{Tr})^{0.3}\right]_{\mathrm{L}}^{\gamma_{1 / 2}}\left(\text { in } \mathrm{kg} / \mathrm{ms}^{2}\right)
$$

eqn. (16), the work form of the new equation, involves only $\mathrm{K}_{\mathrm{J}},{ }_{\mathrm{L}}^{\gamma}, \mathrm{T}, \mathrm{T}_{\mathrm{c}}$ and $\mathrm{V}$.

\section{RESULTS AND DISCUSSIONS}

The new equation, Eqn. (14) or its work form, Eqn. (16) requires molar volume data and critical temperature data in addition to the ratio of liquid heat capacities, while the equation of Srivastava and Berkowitz ${ }^{7}$ requires molar volume and ultrasonic velocity data and, of course the Jacobson constant is required in the newly derived equation. Eqn. (14), proposed in this work, permits evaluation of internal pressure from the Jacobson constant, the Srivastava-Berkowitz group parameter and molar volume data. The equation can readily be extended to multicomponent mixtures.

Internal pressure values were computed for nineteen liquids through the proposed equation, Eqn. (14) and through the Srivastava and Berkowitz equation, Eqn. (3). As shown in Table 1, the proposed model is capable of predicting internal pressure of pure liquids with an average absolute deviation of $4.24 \%$ in the predicted internal pressure values compared to those by the Srivastava-Berkowitz equation. 
Table 1. Internal Pressure Values of Liquids at 298.15K using the Proposed Method

\begin{tabular}{|c|c|c|c|c|c|c|c|c|}
\hline \multirow{3}{*}{$\begin{array}{l}\dot{z} \\
\dot{n}\end{array}$} & \multirow[t]{3}{*}{ Liquid } & 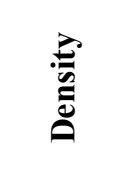 & 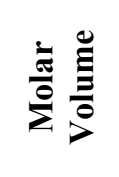 & 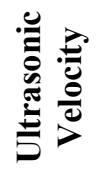 & 胥 & 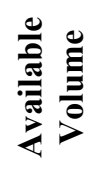 & 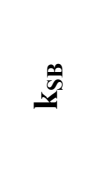 & 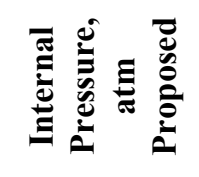 \\
\hline & & $\mathrm{x} 10^{-3}$ & $\times 10^{6}$ & & & $\mathrm{x} 10^{6}$ & & \\
\hline & & $\mathrm{kg} / \mathrm{m}^{3}$ & $\begin{array}{c}\mathrm{m}^{3} / \mathrm{kg} \\
\cdot \mathrm{mol}\end{array}$ & $\mathrm{ms}^{-1}$ & $\mathrm{~K}$ & $\begin{array}{c}\mathrm{m}^{3} / \mathrm{kg} \\
\cdot \mathrm{mol}\end{array}$ & {$[3]$} & Eqn. Eq.(21) \\
\hline 1 & n-Hexane & 0.6551 & 131.55 & 1083 & 507.4 & 30.70 & 3.64 & 2157.59 \\
\hline 2 & n-Heptane & 0.6799 & 147.38 & 1133 & 540.2 & 31.54 & & 2175.63 \\
\hline 3 & n-Octane & 0.6988 & 163.47 & 1170 & 568.8 & 32.65 & & 2164.90 \\
\hline 4 & 2,2,4-Trimethylpentane & 0.6878 & 166.08 & 1077 & 543.9 & 35.22 & & 1991.09 \\
\hline 5 & n-Nonane & 0.7148 & 179.43 & 1212 & 594.6 & 33.81 & & 2142.58 \\
\hline 6 & n-Decane & 0.7267 & 195.80 & 1222 & 617.6 & 35.13 & & 2107.67 \\
\hline 7 & n-Dodecane & 0.7460 & 228.34 & 1288 & 658.3 & 37.13 & & 2032.91 \\
\hline 8 & n-Tetradecane & 0.7599 & 258.43 & 1302 & 694.0 & 40.06 & & 1974.35 \\
\hline 9 & n-Hexadecane & 0.7713 & 293.59 & 1322 & 717.0 & 43.73 & & 1856.44 \\
\hline 10 & Benzene & 0.8742 & 89.35 & 1304 & 562.1 & 18.13 & 4.00 & 3198.86 \\
\hline 11 & Toluene & 0.8627 & 106.81 & 1309 & 591.7 & 20.26 & & 2982.27 \\
\hline 12 & m-Xylene & 0.8602 & 123.42 & 1326 & 617.0 & 22.17 & & 2813.42 \\
\hline 13 & Mesitylene & 0.8615 & 139.52 & 1346 & 637.3 & 24.05 & & 3020.73 \\
\hline 14 & 1-Propanol & 0.8000 & 75.12 & 1204 & 536.7 & 16.22 & 3.29 & 4176.97 \\
\hline 15 & 2-Propanol & 0.7812 & 76.93 & 1141 & 508.3 & 17.91 & & 3744.07 \\
\hline 16 & 1-Butanol & 0.8059 & 91.98 & 1240 & 562.9 & 18.63 & & 3805.05 \\
\hline 17 & 2-Methyl-1-propanol & 0.7932 & 92.86 & 1191 & 536.0 & 20.09 & & 3493.08 \\
\hline 18 & 1-Pentanol & 0.8112 & 108.67 & 1277 & 586.0 & 20.87 & & 3521.18 \\
\hline 19 & 1-Heptanol & 0.8179 & 141.76 & 1330 & 633.0 & 24.65 & & 3163.43 \\
\hline
\end{tabular}

Density and ultrasonic velocity data have been taken from the literature [12]

Table 2. Estimated Internal Pressure Values at 298.15 K Through the Two Methods

(The Srivastava - Berkowitz Method and the Newly Proposed Method)

\begin{tabular}{|c|c|c|c|c|c|c|c|c|c|}
\hline \multirow{4}{*}{$\begin{array}{l}\dot{z} \\
\dot{s}\end{array}$} & \multirow{4}{*}{ Liquid } & \multirow{4}{*}{ 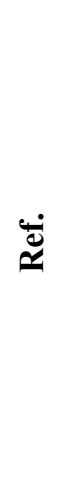 } & 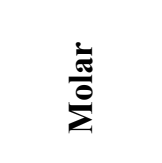 & 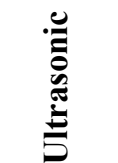 & $\frac{\frac{0}{0}}{\frac{\pi}{\pi / \pi}}$ & تే & $\underline{0}$ & \multicolumn{2}{|c|}{ 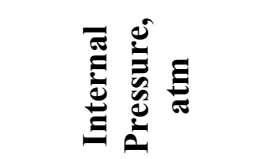 } \\
\hline & & & $\begin{array}{l}\stackrel{\mathscr{E}}{E} \\
\frac{0}{0}\end{array}$ & $\begin{array}{l}\frac{3}{0} \\
\frac{0}{0} \\
\frac{0}{0}\end{array}$ & $\frac{\stackrel{\mathscr{E}}{\mathrm{E}}}{\stackrel{0}{\mathrm{\theta}}}$ & $\frac{\frac{\bar{d}}{\pi}}{\frac{\bar{E}}{\pi}}$ & $\frac{\frac{\bar{d}}{\pi}}{\frac{\bar{\sigma}}{\pi}}$ & 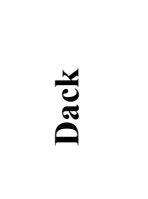 & 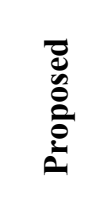 \\
\hline & & & $\times 10^{6}$ & & $\mathrm{x} 10^{6}$ & & & & Eqn. \\
\hline & & & $\mathrm{m}^{3} / \mathrm{kgmol}$ & $\mathrm{ms}^{-1}$ & $\mathrm{~m}^{3} / \mathrm{kgmol}$ & & & [4] & \\
\hline 1 & Acetone & [13] & 74.05 & 1150.0 & 17.24 & 1.699 & 4.195 & 3323.85 & 3019.0 \\
\hline 2 & Acetonitrile & {$[13]$} & 52.81 & 1288.0 & 11.08 & 1.657 & 4.142 & 3839.97 & 4584.8 \\
\hline 3 & Benzene & [13] & 89.40 & 1306.5 & 18.13 & 1.620 & 4.100 & 3736.75 & 3445.3 \\
\hline 4 & tert-Butyl alcohol & [14] & 94.91 & 1123.2 & 22.21 & 2.019 & 4.573 & 3344.49 & 2237.7 \\
\hline 5 & Carbon disulphide & {$[15]$} & 60.63 & 1140.9 & 26.08 & 1.638 & 4.119 & 3716.10 & 4157.1 \\
\hline 6 & Carbontetrachloride & [13] & 97.06 & 927.0 & 19.95 & 1.535 & 3.987 & 3402.30 & 2938.2 \\
\hline 7 & Choloroform & [13] & 80.75 & 983.0 & 17.44 & 1.692 & 4.187 & 3645.91 & 3077.0 \\
\hline 8 & Ethylene glycol & {$[14]$} & 55.91 & 1655.9 & 9.49 & 1.493 & 3.932 & 4954.80 & 5884.2 \\
\hline 9 & n-Hexane & {$[16]$} & 31.59 & 1076.4 & 31.20 & 1.493 & 3.932 & 2357.66 & 1989.7 \\
\hline 10 & 1-Propanol & [14] & 75.15 & 1216.3 & 16.22 & 1.528 & 3.978 & 2840.75 & 3441.7 \\
\hline 11 & 2-Propanol & [17] & 60.68 & 1204.8 & 14.12 & 1.647 & 4.129 & 2766.43 & 3622.8 \\
\hline 12 & Toluene & {$[18]$} & 106.88 & 1303.7 & 20.26 & 1.314 & 3.689 & 3501.39 & 3220.8 \\
\hline & & & & & Avera & absolute & ercenta & deviation & 12.06 \\
\hline
\end{tabular}


Table 2 contains the calculated ${ }_{\mathrm{L}}^{\gamma}$ and $\mathrm{k}_{\mathrm{SB}}$ values as well as the computed internal pressure values through the use of Srivastava-Berkowitz equation and the proposed equation. When compared with literature values ${ }^{7}$ for twelve liquids considered in Table 2 , the average absolute deviations in the predicted internal pressure values are $10.74 \%$ (the SrivastavaBerkowitz equation) and $12.06 \%$ (proposed equation), showing the performancecharacteristics of these equations. The purely predictive nature of the proposed equation has to be considered at this juncture.

The applicability of the proposed equation in predicting the internal pressures of liquid mixtures was ascertained by using the data of five liquid mixtures, trichloroethylene + benzene / carbon tetrachloride / chloroform / toluene / p-xylene for which the results on the Srivastava-Berkowitz equation are available ${ }^{11}$; nearly identical results were obtained. The calculated and predicted internal pressure values were obtained. The calculated and predicted internal pressure values through the Srivastava-Berkowitz equation and the new equation of this study are given in Table 3. The average absolute percentage deviation in predicted internal pressure values for the five liquid mixtures shown in Table 3, varies from 0.29 to $1.91 \%$ in comparison with those given by Srivastava-Berkowitz equation ${ }^{7}$.

Table 3. Internal Pressure from various methods at $303.15 \mathrm{~K}$.

\begin{tabular}{|c|c|c|c|c|c|c|c|c|c|}
\hline \multicolumn{10}{|c|}{ Trichloroethylene - Benzene } \\
\hline $\mathrm{x}_{1}$ & 0.1112 & 0.2565 & 0.3679 & 0.5456 & 0.6064 & 0.7435 & 0.9459 & & \multirow{4}{*}{$\begin{array}{c}\text { Average } \\
\text { absolute } \\
\text { percentage } \\
\text { deviation } \\
0.29\end{array}$} \\
\hline Srivastava-Berkowitz Eq., $\pi_{\mathrm{i}}(\mathrm{atm})$ & 3651.1 & 3644.3 & 3631.7 & 3629.2 & 3630.9 & 3635.8 & 3624.4 & & \\
\hline Newly Proposed Eq. $\pi_{\mathrm{i}}(\mathrm{atm})$ & 3642.5 & 3634.5 & 3630.1 & 3624.2 & 3622.5 & 3619.5 & 3601.1 & & \\
\hline Absolute percentage deviation & 0.24 & 0.27 & 0.04 & 0.14 & 0.23 & 0.45 & 0.64 & & \\
\hline \multicolumn{10}{|c|}{ Trichloroethylene - Toluene } \\
\hline $\mathrm{x}_{1}$ & 0.1256 & 0.2537 & 0.6721 & 0.7233 & 0.9395 & & & & \multirow{4}{*}{$\begin{array}{c}\text { Average } \\
\text { absolute } \\
\text { percentage } \\
\text { deviation } \\
0.73\end{array}$} \\
\hline Srivastava-Berkowitz Eq., $\pi_{\mathrm{i}}(\mathrm{atm})$ & 3510.0 & 3551.9 & 3584.0 & 3593.9 & 3628.3 & & & & \\
\hline Newly Proposed Eq. $\pi_{\mathrm{i}}(\mathrm{atm})$ & 3561.4 & 3596.4 & 3596.1 & 3599.4 & 3612.5 & & & & \\
\hline Absolute percentage deviation & 1.46 & 1.25 & 0.34 & 0.15 & 0.44 & & & & \\
\hline \multicolumn{10}{|c|}{ Trichloroethylene - p-Xylene } \\
\hline $\mathrm{x}_{1}$ & 0.2137 & 0.2299 & 0.3990 & 05717 & 0.6011 & 0.7598 & & & \multirow{4}{*}{$\begin{array}{c}\text { Average } \\
\text { absolute } \\
\text { percentage } \\
\text { deviation } \\
1.91\end{array}$} \\
\hline Srivastava-Berkowitz Eq., $\pi_{\mathrm{i}}(\mathrm{atm})$ & 3400.1 & 3413.2 & 3454.0 & 3490.6 & 3501.3 & 3552.6 & & & \\
\hline Newly Proposed Eq. $\pi_{\mathrm{i}}(\mathrm{atm})$ & 3491.1 & 3494.1 & 3524.2 & 3553.8 & 3558.9 & 3584.7 & & & \\
\hline Absolute percentage deviation & 2.68 & 2.37 & 2.03 & 1.81 & 1.64 & 0.90 & & & \\
\hline \multicolumn{10}{|c|}{ Trichloroethylene - Chloroform } \\
\hline $\mathrm{x}_{1}$ & 0.2299 & 0.3399 & 0.4665 & 0.6863 & 0.7221 & 0.8367 & 0.9303 & & \multirow{4}{*}{$\begin{array}{c}\text { Average } \\
\text { absolute } \\
\text { percentage } \\
\text { deviation } \\
1.63\end{array}$} \\
\hline Srivastava-Berkowitz Eq., $\pi_{\mathrm{i}}(\mathrm{atm})$ & 3592.8 & 3599.2 & 3604.9 & 3631.3 & 3629.4 & 3632.2 & 3647.6 & & \\
\hline Newly Proposed Eq. $\pi_{\mathrm{i}}(\mathrm{atm})$ & 3504.5 & 3521.6 & 3540.7 & 3576.8 & 3578.2 & 3593.7 & 3608.9 & & \\
\hline Absolute percentage deviation & 2.46 & 2.15 & 1.78 & 1.50 & 1.41 & 1.06 & 1.06 & & \\
\hline \multicolumn{10}{|c|}{ Trichloroethylene - Carbon tetrachloride } \\
\hline $\mathrm{x}_{1}$ & 0.1343 & 0.2258 & 0.3196 & 0.4385 & 0.6352 & 0.7258 & 0.8319 & 0.9439 & \multirow{4}{*}{$\begin{array}{c}\text { Average } \\
\text { absolute } \\
\text { percentage } \\
\text { deviation } \\
0.95\end{array}$} \\
\hline Srivastava-Berkowitz Eq., $\pi_{\mathrm{i}}(\mathrm{atm})$ & 3381.9 & 3306.5 & 3350.8 & 3403.4 & 3485.5 & 3526.3 & 3573.3 & 3622.6 & \\
\hline Newly Proposed Eq. $\pi_{\mathrm{i}}(\mathrm{atm})$ & 3262.7 & 3298.1 & 3334.8 & 3382.7 & 3464.2 & 3502.9 & 3549.1 & 3595.6 & \\
\hline Absolute percentage deviation & 3.52 & 0.26 & 0.48 & 0.61 & 0.61 & 0.66 & 0.68 & 0.74 & \\
\hline
\end{tabular}




\section{CONCLUSION}

A new method was developed for the prediction of internal pressure of pure liquids and liquid mixtures. This theoretical model combines the Free Length Theory of Jacobson and van der Waals' equation of state. It requires only the molar volume data in addition to the ratio of heat capacities and critical temperature. The model is simple and reliably accurate. The predictive ability of the new equation was tested by comparing the internal pressure values obtained through this equation with those of the Srivastava-Berkowitz equation.

\section{REFERENCES}

[1] J. H. Hildebrand, R. L. Scott, Solubility of Non-electrolytes, III Edn., Reinhold Publ. Corpn., New York, (1950) p.121.

[2] J. H. Hildebrand, R. L. Scott, Regular Solutions, Prentice-Hall, Englewood Cliffs, New Jersey, 1962.

[3] T. W. Richards, Chem. Rev. 2 (1925) 315.

[4] M. R. Dack, J. Chem. Soc. Rev. 4(2) (1975) 211.

[5] G. Z. Kortum, Electrochem. 42 (1936) 287.

[6] A. F. M.Barton, J. Chem. Edu. 48(3) (1971) 156.

[7] S. C. Srivastava, N. Berkowitz, Can. J. Chem. 41 (1963) 1787.

[8] B. Jacobson, Acta Chem. Scand. 6 (1952) 1485.

[9] B. Jacobson, J. Chem. Phys. 20 (1952) 927.

[10] N. Santhi, "Ultrasonic velocity, free volume and internal pressure of liquids and liquid mixtures" Ph.D. Thesis in Chemistry, Annamalai University, Annamalainagar (1998).

[11] A. P. Srivastava, S. N. Dubey, Acoustics Lett. 8(11) (1985) 191.

[12] T. M. Aminabhavi, et al., Indian J. Technol. 31 (1993) 27.

[13] R. J. Fort, and W. R. Moore, Trans. Faraday Soc. 61 (1965) 2102.

[14] N. Manohara Murthy, G. Nagabhushanam, Acustica 54 (1984) 225.

[15] V. Hyder Khan, G. Ravindranath, J. Anjaneyulu, Acustica 71 (1990) 14.

[16] G. Nagabhushanam, N. Manohara Murthy, Acustica 65 (1988) 295.

[17] R. L. Blokhra, R. Kumar, Acustica 68 (1989) 161.

[18] K. Malakondaiah, S. V. Subramanyam, Acoustics Lett. 11(3) (1987) 48. 